\title{
Las prácticas pedagógicas en la universidad: sujetos y saberes
}

\section{The Pedagogical Practices in the University: Subjects and Knowledge}

\section{Práticas pedagógicas na universidade: disciplinas e conhecimentos}

\author{
Jesús Manuel Rodelo Pérez \\ Universidad Autónoma de Occidente, Sinaloa, México \\ jmrodelo@gmail.com \\ http://orcid.org/0000-0002-2705-2519
}

\section{Resumen}

El objetivo de este trabajo fue caracterizar y explicar los conocimientos y saberes presentes en las prácticas de enseñanza de los docentes universitarios. A partir del análisis de las narrativas de los profesores en torno a sus conocimientos, saberes y experiencias en el campo de la docencia, se buscó aprehender las maneras en que los profesores universitarios construyen dichos saberes. Con base en una metodología cualitativa se aplicaron 40 entrevistas abiertas no estructuradas a docentes de la Universidad Autónoma de Occidente de la Unidad Regional Culiacán. La muestra se conformó con base en un listado de los atributos esenciales de los sujetos objeto de estudio, siguiendo el procedimiento que Goetz y LeCompte (1988) denominan como una selección basada en criterios.

En los resultados, los docentes reconstruyen sus actos docentes en un ejercicio reflexivo; es a partir de ello que se manifiestan los saberes disciplinarios como ejes de su enseñanza, mientras que los saberes pedagógicos permanecen al margen. Estos se hacen más bien presentes en los recursos y dispositivos con las cuales los profesores, en el ámbito de su acción pedagógica, desarrollan estrategias de acción que facilitan los procesos de enseñanzaaprendizaje. 


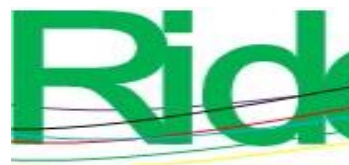

Revista Iberoamericana para la Investigación y el Desarrollo Educativo

ISSN $2007-7467$

Palabras clave: conocimientos y saberes docentes, docencia universitaria, prácticas pedagógicas, práctica reflexiva, saberes profesionales.

\section{Abstract}

The objective of this work was to characterize and explain the pedagogical knowledge present in the teaching practices of university teachers. From the analysis of the narratives of the professors around their knowledge and experiences in the field of teaching, it was sought to apprehend the ways in which university professors construct such knowledge. Based on a qualitative methodology, 40 unstructured open interviews were applied to teachers from the Autonomous University of the Western Region of Culiacán. The sample was based on a list of the essential attributes of the subjects under study, following the procedure that Goetz and LeCompte (1988) call a selection based on criteria.

In the results, the teachers reconstruct their teaching acts in a reflective exercise in which the disciplinary knowledge is manifested as the axis of their teaching, while the pedagogical knowledge remains on the margin, making itself present in the resources and devices with which the teachers, in the field of their pedagogical action, develop strategies of action that facilitate the teaching-learning processes.

Keywords: teaching knowledge and knowledge, university teaching, pedagogical practices, reflexive practice, professional knowledge.

\section{Resumo}

O objetivo deste trabalho foi caracterizar e explicar o conhecimento e o conhecimento presentes nas práticas de ensino de professores universitários. A partir da análise das narrativas dos professores em torno de seus conhecimentos, conhecimentos e experiências no campo do ensino, buscou-se apreender as maneiras pelas quais os professores universitários constroem esse conhecimento. Com base em metodologia qualitativa, 40 entrevistas abertas não estruturadas foram aplicadas a professores da Universidade Autônoma do Oeste da Unidade Regional de Culiacán. A amostra foi baseada em uma lista dos atributos essenciais dos sujeitos em estudo, seguindo o procedimento que Goetz e LeCompte (1988) chamam de seleção com base em critérios. 


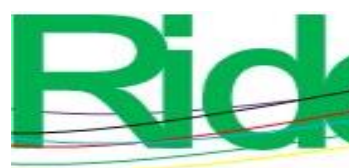

Revista Iberoamericana para la Investigación y el Desarrollo Educativo

ISSN $2007-7467$

Nos resultados, os professores reconstroem suas ações de ensino em um exercício reflexivo; É a partir disso que o conhecimento disciplinar se manifesta como eixos de seu ensino, enquanto o conhecimento pedagógico permanece à margem. Elas estão presentes nos recursos e dispositivos com os quais os professores, no âmbito de sua ação pedagógica, desenvolvem estratégias de ação que facilitam os processos de ensino-aprendizagem.

Palavras-chave: ensino de conhecimento e conhecimento, ensino universitário, práticas pedagógicas, prática reflexiva, conhecimento profissional.

Fecha Recepción: Junio 2019

Fecha Aceptación: Octubre 2019

\section{Introducción}

Tardif en 1991 publicó un trabajo pionero sobre el saber docente que dio con una idea que corría subrepticiamente a la espera de su revelación. Ludke (2010) comenta al respecto:

La fina percepción y la descripción cuidadosa de esta propiedad específica de los profesores, su saber, abrieron una perspectiva de estudios y de postura, incluso en relación con la profesión docente, con repercusión sobre la producción académica, los programas de los cursos de formación de profesores y la propia legislación respecto de estos cursos y la carrera docente (p. 194).

Esta importante contribución al estudio de la profesión docente se ha constituido en una animosa influencia en los profesores al propugnar que detentan un saber específico propio. Tardif (2009) describe este saber como "plural, formado por una amalgama, más o menos coherente, de saberes procedentes de la formación profesional y disciplinarios, curriculares y experienciales" (p. 299). En este trabajo nos interesa conocer cómo el docente universitario construye su saber con base en los conocimientos adquiridos en su formación disciplinar y la experiencia en su vida académica. Consideramos que los saberes producto de su quehacer en el aula le permiten al docente comprender y dominar su práctica.

$\mathrm{Al}$ inicio de su carrera, los profesores universitarios son profesionales que sustentan su práctica en los conocimientos de su disciplina. La institución educativa es el lugar donde llevan cabo su socialización profesional. Con el paso del tiempo y el accionar en el aula, integran a su conocimiento especializado un conjunto de herramientas constituidas por conocimientos, experiencias, actitudes y valores que adquiere día a día en el entorno educativo. Es así como en la vida cotidiana laboral se apropia de los rudimentos del oficio: los 


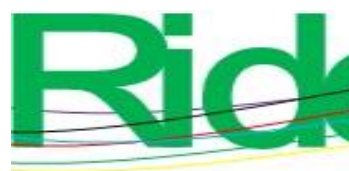

Revista Iberoamericana para la Investigación y el Desarrollo Educativo

ISSN $2007-7467$

saberes, los discursos propios del trabajador docente, las tradiciones de un centro educativo determinado, diferente a otro. De esa manera, se conforma la cultura del gremio en el seno de la institución escolar. Al respecto, Fierro, Fortoul y Rosas (2002) señalan:

Es el organismo vivo que explica que la escuela no sea solamente la suma de individuos y acciones aisladas, sino una construcción cultural en la que cada maestro aporta sus intereses, sus habilidades, sus proyectos personales y sus saberes a una acción educativa común (p. 76).

Es reducido el conocimiento que se tiene con respecto a las prácticas pedagógicas y los saberes que los docentes ponen en juego en su trabajo cotidiano en las aulas universitarias. Surge entonces la necesidad de resignificar estos saberes que en la actualidad han tomado gran relevancia en la investigación de las prácticas de enseñanza y aprendizaje, lo cual conlleva una serie de consideraciones. Y entre estas destaca la siguiente:

Reconocer el papel activo del profesor y las estrategias que utiliza en su práctica, por cuanto es a través de estas como el profesor pone en juego un conocimiento que está vinculado no solamente a un saber disciplinar, sino que está vinculado también a teorías educativas, modelos pedagógicos, paradigmas de aprendizaje, y estos se relacionan también con los planes de estudio, el currículo y la concepción de educación (Jiménez y Rendón, 2014, p. 140).

De acuerdo con Londoño (2014, p. 26), "el profesor universitario, desde su experiencia; desde la posibilidad de pensar, reflexionar y potenciar su práctica; y de capitalizar sus conocimientos, aciertos y desaciertos, construye un saber". De ahí la importancia de conocer, además de las competencias disciplinarias de los profesores, las competencias pedagógicas inherentes a la labor del docente, además de la enseñanza, la planeación y evaluación de los aprendizajes de los estudiantes.

Teniendo en cuenta lo anterior, la pregunta principal de nuestra investigación fue la puesta a continuación: ¿De qué manera los docentes universitarios construyen sus saberes con base en los conocimientos adquiridos en su formación disciplinar y la experiencia en su vida académica? 


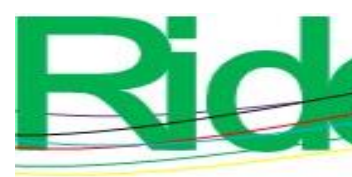

Revista Iberoamericana para la Investigación y el Desarrollo Educativo ISSN $2007-7467$

\section{Objetivo}

El objetivo general de este trabajo fue caracterizar y explicar los conocimientos y saberes presentes en las prácticas de enseñanza de los docentes universitarios. A partir del análisis de las narrativas de los profesores en torno a sus conocimientos, saberes y experiencias en el campo de la docencia, se buscó aprehender cómo los profesores universitarios construyen dichos saberes. La docencia universitaria como campo de estudio implica una mirada sobre las instituciones del nivel superior y su labor en la formación de profesionales, por eso consideramos pertinente investigar el trabajo docente y reflexionar en torno a lo subyacente en sus prácticas educativas. Es en ellas donde el docente aplica sus saberes disciplinarios, teóricos y pedagógicos que se conjugan con una peculiar idea de su concepción de la educación.

\section{Conocimientos y saberes docentes}

El término saber tiene diversas acepciones. Destaca aquí, sin embargo, la definición de Beillerot (citado en Altet, 2012, P. 41), quien la formula de la siguiente manera: "Lo que para un sujeto está adquirido, construido, elaborado gracias al estudio o la experiencia”. Y para una mayor precisión conceptual es igualmente importante distinguir entre información, saber y conocimiento. Legroux (citado en Altet, 2012, p. 41) señala que la información es "exterior al sujeto y de orden social"; el conocimiento es "integrado por el sujeto, y es de orden personal". Mientras que, para el propio Altet (2012), "el saber se construye en la interacción entre conocimiento e información, entre sujeto y entorno, dentro y a través de la mediación" (p. 42).

Los conocimientos docentes comprenden un vasto territorio cuya cartografía varía según las diversas disciplinas que se ocupan de su estudio. En ese sentido existe una pluralidad de conocimientos docentes. Tenemos, entonces, conocimientos que son planeados por los docentes y que guían las acciones de enseñanza, pero también aquellos producto de la experiencia del maestro: las improvisaciones de situaciones inesperadas en clase, las rutinas que permiten atender grupos numerosos y otras situaciones que no aparecen en ningún manual de didáctica. De acuerdo con Altet (2012), investigadores del campo de las ciencias cognitivas han desarrollado trabajos sobre el pensamiento de los maestros, entre ellos los modelos de procesamiento de la información y la clasificación de Anderson sobre los tipos de saberes: el declarativo (saber qué), el procedimental (saber cómo) y el condicional o contextual (saber 


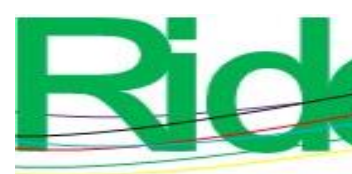

Revista Iberoamericana para la Investigación y el Desarrollo Educativo

ISSN $2007-7467$

cuándo y dónde). Los cuales hacen posible distinguir los diferentes modos en que asumen los conocimientos docentes. También permiten describir las formas que adquieren los conocimientos en la etapa de planeación y de esta manera poder explicar los mecanismos del trabajo docente en una acción rutinaria. Altet (2012) explica las consecuencias del enfoque cognitivista. Por un lado, nos dice, este enfoque ha permitido delimitar los procesos de decisión de los docentes. Por el otro:

No permite conocer la naturaleza de los conocimientos subyacentes en los procesos de toma de decisiones. No parece que los algoritmos, los métodos heurísticos, las normas de acción y los planes racionales preestablecidos puedan rendir cuentas de la adaptabilidad del maestro durante la acción frente a una situación pedagógica siempre singular, incierta y muy compleja. El maestro no puede decidir por sí mismo (Altet, 2012, p. 43).

Tochon (citado en Altet, 2012, p. 43), consigna que el docente en su trabajo utiliza razonamientos maleables, analógicos, intuitivos o "abductivos", obtenidos de su experiencia, la cual posibilita la producción de ideas nuevas en situaciones inesperadas. Shön (1987), en su modelo sobre la práctica reflexiva, describe lo anterior como la reflexión en la acción, con la cual se clarifican situaciones de la práctica que son inciertas, singulares o conflictivas. En este tipo de prácticum se ubican aquellas situaciones en las que el conocimiento profesional existente no basta ni se acomoda a cada caso.

\section{Conceptualizando el saber}

De acuerdo con Tardif y Gauthier (2012), en la cultura de la modernidad se conceptualizan tres formas de saber: 1) el sujeto y la representación, 2) el juicio y el discurso asertivo y 3) el argumento y la discusión. La primera concepción alude a la subjetividad, "a un tipo particular de certeza subjetiva producida por el pensamiento racional" (p. 322). Esta concepción se opone a certezas subjetivas fundadas como la fe, la creencia o el prejuicio. De acuerdo con los mismo autores, la certeza subjetiva propia del saber adquiere dos formas: $a$ ) "la forma de una intuición intelectual a través de la cual se puede buscar y aprehender inmediatamente una verdad" (p. 323), ejemplo de esto serían ciertas verdades matemáticas o lógicas (el todo es mayor que la parte); y b) "la forma de una representación intelectual, 


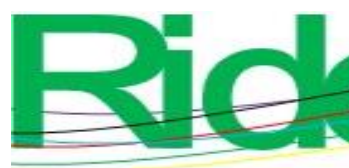

Revista Iberoamericana para la Investigación y el Desarrollo Educativo

ISSN $2007-7467$

resultante de una cadena de razonamientos o de una inducción” (p. 323). Este tipo de certeza subjetiva, además, lo relacionamos con lo que Dewey (1989) describe como reflexión:

La reflexión no implica tan solo una secuencia de ideas, sino una consecuencia, esto es, una ordenación consecuencial en la que cada una de ellas determina la siguiente como su resultado, mientras que cada resultado, a su vez, apunta y remite a los que la precedieron. Los fragmentos sucesivos de un pensamiento reflexivo surgen unos de otros y se apoyan mutuamente; no aparecen y desaparecen súbitamente en una masa confusa y alborotada (p. 22).

Como resultado de un proceso de razonamiento, la representación es mediata, a diferencia de la intuición que es inmediata, por lo que, afirman Tardif y Gauthier (2012), aquí la subjetividad es considerada como "el lugar del saber", y por lo tanto, "saber algo es poseer una certeza subjetiva racional" (p. 151).

Para Tardif y Gauthier (2012) el juicio verdadero es un saber, entendiéndolo como "el discurso que afirma con razón alguna cosa acerca de otra cosa” (p. 324). Estamos aquí en el terreno del discurso, en la aserción. En esta concepción solo los discursos referidos a hechos pueden ser catalogados como un saber en sentido estricto, es decir, se limita a juicios de facto y deja fuera a los juicios de valor y las vivencias. De esta manera, el juicio sería también "un lugar del saber".

En su tercera concepción del saber, Tardif y Gauthier (2012) relacionan a este con el argumento y la discusión. Dicha manera de entender denomina saber "a la actividad discursiva que consiste en intentar validar una proposición o acción con la ayuda de argumentos y de operaciones lingüísticas y discursivas (lógicas, retóricas, dialécticas, empíricas, etc.)” (p. 326). La argumentación, señalan Tardif y Gauthier (2012), es el lugar del saber, pues saber una cosa no es solo emitir un juicio verdadero sobre una cosa, también se necesita argumentar el por qué un juicio es verdadero, lo cual nos lleva a la necesidad de la presencia del otro sujeto receptor de ese argumento, convirtiéndose de esta manera en un intercambio discursivo, en una comunicación intersubjetiva. Así, pues, siguiendo a este par de autores, los criterios de validez argumentativa ya no se limitan a la aportación de razones que demuestren la validez de un discurso, sino que "responden a la idea de la armonía comunicacional en el seno de una comunidad de discusión. En ese sentido, los llamados juicios de valor pueden ser objeto de consensos racionales" (Tardif y Gauthier, 2012, p. 237). 


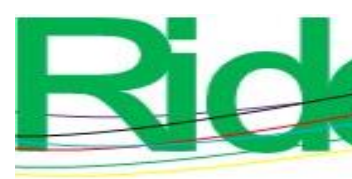

Revista Iberoamericana para la Investigación y el Desarrollo Educativo

ISSN $2007-7467$

Las tres concepciones presentadas anteriormente, pese a sus diferencias, confluyen en conjuntar la naturaleza del saber con exigencias de racionalidad. Es así como no basta la presentación adecuada de un discurso, sino la presencia de razones o motivos que validen tal discurso. De igual manera, es necesario que el sujeto, además de saber hacer una cosa, explique por qué realiza las cosas de cierta forma y no de otra.

En ese sentido, para efectos de investigar y relacionar la noción de saber con la idea de racionalidad, Tardif y Gauthier (2012) plantean los elementos que se deben tomar en cuenta respecto a los saberes de los docentes:

1. De ahora en adelante llamaremos saber únicamente a los pensamientos, las ideas, los juicios, discursos y argumentos que responden a ciertas exigencias de racionalidad. Yo hablo o actúo racionalmente cuando soy capaz de motivar mi discurso o mi acción (con la ayuda de razonamientos, declaraciones, procedimientos, etc.) frente a otro actor que me pregunta acerca de su pertinencia, su valor, etc.

2. Cuando el locutor o el actor al que nos dirigimos, para motivar sus pensamientos, juicios, discurso y actos, es capaz de exponer unas razones, sin importarle su naturaleza o su contenido de verdad, entonces podemos decir que las exigencias anteriormente señaladas se han respetado.

3. Al proceder así, evitaremos imponer a los actores un modelo preconcebido de lo que es racional o no; más bien partiremos de lo que ellos consideran racional, y nos esforzaremos en sacar a la luz sus propias exigencias de racionalidad y su propia concepción del saber.

4. El mejor método del que dispone el locutor o el actor para acceder a las exigencias de racionalidad consiste en preguntar (o preguntarse) sobre el porqué; es decir, preguntarse sobre las causas, las razones, los motivos de su discurso o de su acción.

5. (...) Una de las principales estrategias de investigación que coincide con esta visión del saber consiste en observar a los actores y/o hablar con ellos, pero preguntándoles sobre sus razones para actuar o discurrir; es decir, cuestionándolos sobre los saberes en los que se apoyan para actuar o discurrir (pp. 328-329). 


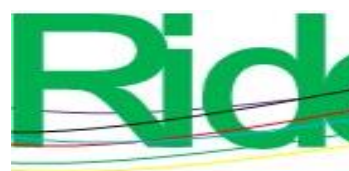

2

\begin{abstract}
(a)
\end{abstract}
ISSN 2007-7467

Estos elementos de la investigación sobre lo saberes docentes puntualizan las características de la propuesta de Tardif y Gauthier (2012), alejada de un modelo rígido que impide llegar a las razones y motivos que orientan y justifican sus acciones. Los sujetos están dotados de racionalidad, es decir, tienen la capacidad de actuar, de hablar y de pensar, capacidad con la cual ordenan las razones que orientan su práctica. Constituye una habilidad esencial de los sujetos con la cual responden a las exigencias de racionalidad. Esta habilidad, señalan una vez más Tardif y Gauthier (2012), no debe ser abordada con una idea preconcebida, positivista; "por el contrario, hay que aceptar el hecho de que la competencia de los actores sociales proviene de una racionalidad dinámica, fluida, que no responde necesariamente a los cánones del pensamiento lógico y científico" (p. 334).

Llegado a este punto no está de más explicitar que en este trabajo se siguió el modelo de Tardif y Gauthier (2012) para identificar los saberes de los docentes universitarios en los discursos emitidos entorno a sus prácticas pedagógicas.

\section{Enfoque metodológico}

Con base en una metodología cualitativa, se aplicaron 40 entrevistas abiertas no estructuradas a docentes de la Universidad Autónoma de Occidente, Unidad Regional Culiacán. La muestra se conformó con base en un listado de los atributos esenciales de los sujetos objeto de estudio, siguiendo el procedimiento que Goetz y LeCompte (1988) denominan como una selección basada en criterios. Se buscó que los docentes pertenecieran a diferentes licenciaturas con la finalidad de contemplar los diversos campos del conocimiento. Se diseñó un guión para la entrevista que recogió los principales temas de la investigación. La entrevista tuvo un tono conversacional, el guión sirvió como una guía para abordar las áreas de interés. Elegimos la entrevista cualitativa a razón de lo que Tardif y Gauthier (2012) mencionan a continuación:

Una de las principales estrategias de investigación que coincide con esta visión del saber consiste en observar a los actores y/o hablar con ellos, pero preguntándoles sobre sus razones para actuar o discurrir; es decir, cuestionándolos sobre los saberes en los que se apoyan para actuar o discurrir (p. 330). 


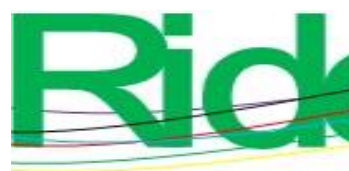

Para la interpretación de los datos recolectados en las entrevistas, el referente metodológico fue la perspectiva de la hermenéutica analógica (Beuchot, 2008). Tomando en cuenta que la información recabada en las entrevistas son textos que "si bien son elaboraciones de sus autores, son parte de las relaciones comunicativas que ellos establecen con otros actores o comunidades de actores, y en esa medida son construcciones sociales que forman parte de la historia de una colectividad profesional” (Bolán, citado en Roldán, 2016, p. 211). Las construcciones desde la perspectiva de los docentes posibilitan comprender sus prácticas, entender la acción como discurso a través de las configuraciones narrativas (Jiménez y Rendón, 2014). En ese sentido, Beuchot (2016) propone una investigación de la educación de corte hermenéutico y analógico. Hermenéutico porque concibe la interacción educativa como un texto que requiere una interpretación de acuerdo con el contexto y los diferentes códigos implícitos en los mensajes. Con respecto a lo analógico, el autor reconviene que en la interpretación no se busca una interpretación exacta, una visión unívoca, antes bien deberá contener una apertura aceptable, cuidando siempre de no caer en un relativismo desmesurado.

\section{Resultados}

Con los datos obtenidos en el trabajo de campo se clasificó la formación inicial de los docentes universitarios, tal y como se describe a continuación: $37.5 \%$ pertenece al campo de las ciencias sociales y humanidades (psicología, derecho, comunicación); $25 \%$ a ingeniería; otro $25 \%$ son licenciados en Administración y Contaduría; y $12.5 \%$ pertenece al área de las ciencias computacionales. La mitad $(50 \%)$ realizó estudios de posgrado, generalmente en las áreas de su formación inicial. Solo dos mencionaron haber estudiado un posgrado en Educación. El total afirmó haber tomado cursos de capacitación (al menos dos durante su trayectoria laboral en la universidad), relacionados con pedagogía, en modalidades de diplomados y la gran mayoría en cursos sueltos. Los contenidos de los cursos, en general, se relacionaban con las competencias, la didáctica y los estilos de aprendizaje. Precisamente uno de los hallazgos encontrados fue la importancia que los docentes otorgan a los cursos de capacitación. En las entrevistas fue posible detectar que los profesores universitarios utilizan diversas estrategias pedagógica impartidas en los cursos. No obstante, muchos de ellos consideran los saberes disciplinarios y la exposición magistral como lo esencial de sus clases. 


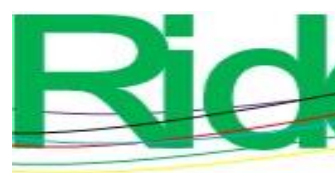

Revista Iberoamericana para la Investigación y el Desarrollo Educativo

ISSN $2007-7467$

La interpretación de las narrativas de los docentes en torno a sus prácticas pedagógicas en la universidad nos permitió construir las categorías e integrarlas en un corpus teórico interpretativo que configura el apartado de los resultados de la investigación. Las categorías se presentan de la manera siguiente: Docencia universitaria, El saber pedagógico del docente universitario, Práctica profesional y docencia y La reflexión como ejercicio permanente.

\section{Docencia universitaria}

Investigar la docencia universitaria como profesión implica analizar los discursos de los profesores, tomando en cuenta que ellos, de origen, no poseen una formación para ejercer la profesión docente. En las entrevistas se pudo advertir que los profesores asumen esta condición y lo manifiestan de una manera consciente. Una docente afirma al respecto:

Nosotros, los maestros universitarios, no tenemos una formación pedagógica y didáctica, o sea, es mínimo. No nos forman para ser maestros realmente nos forman para trabajar, para ser profesionistas, para darle respuesta a la sociedad y los problemas que se suscitan en el mundo laboral

Los profesores del nivel superior, por diferentes causas, encontraron en la docencia una profesión con la cual se encuentran comprometidos también de diferentes maneras. En las narrativas de los docentes se manifiestan diversas trayectorias para llegar a ejercer la docencia. Una ruta detectada es la invitación que profesores universitarios hacen a egresados, con los cuales tienen un tipo de relación afectiva o laboral. En los diversos ejercicios profesionales se establecen relaciones personales que algunas veces inciden en el ingreso a diferentes empleos, en este caso a la docencia universitaria. Otra manera de llegar a la docencia es el trabajo administrativo que se ejerce en una institución educativa, y luego, por diversas circunstancias, se accede al trabajo en el aula.

El salto del ejercicio profesional a la docencia también se da de una manera intencionada. El docente se acerca a la institución con el deseo de ingresar al sistema, ya sea de manera directa presentando solicitudes de ingreso o a través de evaluaciones que las instituciones realizan para la obtención de una plaza. Los estudios de posgrado son además una vía de acceso a la docencia, generalmente como parte de la carrera de docente investigador. Asimismo, le otorgan a los profesionistas que se dedican a la docencia un valor agregado que facilita su inserción en las instituciones educativas. 
El saber pedagógico del docente universitario

Tardif (2009) señala que el análisis del trabajo de los docentes permite explicar su relación con la pedagogía. Para este pensador las investigaciones pedagógicas en general están basadas en abstracciones, dejando de lado situaciones del contexto, tales como el tiempo de trabajo, el número de estudiantes, los recursos disponibles, la materia a impartir y su naturaleza, los condicionantes presentes, las relaciones con los compañeros y con los docentes especialistas, los saberes de los agentes, el control de la administración, etc. Estas abstracciones impiden que el estudio de la pedagogía se sitúe en el contexto más amplio del análisis del trabajo de los docentes, como es pertinente que se realice. De ahí que Tardif (2009) señale que la pedagogía como categoría se debe ubicar en relación con las situaciones de trabajo vividas por los docentes. En consecuencia, dicho autor define la pedagogía como:

El conjunto de los medios empleados por el docente para alcanzar sus objetivos en el ámbito de las interacciones educativas con los alumnos. En otras palabras, desde el punto de vista del análisis del trabajo. La pedagogía es la "tecnología" utilizada por los docentes en relación con su objeto de trabajo (los alumnos), en el proceso del trabajo cotidiano, para obtener un resultado (la socialización y la instrucción) (Tardif, 2009, p. 86).

La tecnicidad es inherente al trabajo, dice Tardif (2009), y por lo tanto enseñar es utilizar una cierta tecnología. Entonces, la pedagogía sería la dimensión instrumental de la enseñanza, la práctica concreta situada en un ambiente de trabajo. Al respecto, afirma este mismo autor: "Así como no existe un trabajo sin técnica, tampoco existe un proceso de enseñanza-aprendizaje sin pedagogía, aunque se manifieste con frecuencia una pedagogía sin reflexión pedagógica" (Tardif, 2009, p. 88). Esta concepción de la pedagogía permite comprender el actuar del docente universitario en el aula y explicar cómo los docentes universitarios generan saber pedagógico en sus prácticas de enseñanza con base en sus experiencias vividas.

Asimismo, en este apartado se retoma el modelo de Tardif y Gauthier (2012) para investigar la presencia de la noción de saber, con la idea de racionalidad, en la narrativa de los profesores. En el segmento discursivo que presentamos a continuación es evidente la existencia de una conciencia cabal de la maestra citada en el sentido de su escasa preparación en el ámbito docente; sin embargo, su trayectoria en la docencia, su experiencia en el aula y la 


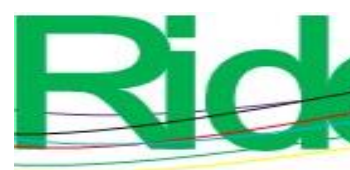

Revista Iberoamericana para la Investigación y el Desarrollo Educativo

ISSN $2007-7467$

capacitación recibida en su centro de trabajo le permite una reflexión en torno a su labor educativa:

D-C-3: Apegarnos a una perspectiva pedagógica, hablarte de Freud o de Piaget o de los grandes clásicos, pues, no, no los tengo presentes en mi clase como para decir que hoy voy a utilizar esta pedagogía o esta técnica, pero sí procuro estar en la innovación, estar tratando de hacer que los estudiantes en la medida de sus posibilidades y de las mías entren en discusiones, entren en dinámicas que les permitan entrar en ese proceso, que sientan ese proceso que están aprendiendo. Y también procuro identificar, también en la medida que puedo, los distintos estilos que tienen los estudiantes para aprender, porque eso es muy importante por parte, creo yo, del profesor, que entienda también cuáles situaciones y cuáles limitaciones también tienen, como las tenemos nosotros como profesor para aprender y enseñar.

En el fragmento anterior se puede observar la concepción que la profesora tiene del trabajo docente desde su propia trayectoria y formación. Ella menciona que ciertas teorías del aprendizaje no están presentes en su práctica; sin embargo, hace alusión a los estilos de aprendizaje de los estudiantes, los cuales valora porque le permiten establecer las diferencias en sus alumnos y actuar en consecuencia. En este caso se presenta un saber relacionado con el argumento y la discusión, de acuerdo con el modelo de Tardif y Gauthier (2012). En el discurso de la docente hay la intención de validar una proposición con argumentos y operaciones lingüísticas y discursivas. La argumentación, mencionan Tardif y Gauthier (2012), es el lugar del saber. En la narrativa de la maestra existe una racionalidad en su argumentación discursiva al exponer los motivos que orientan su práctica pedagógica.

En la misma tónica, Contreras y Pérez (2013) colocan la experiencia en el centro del proceso educativo. Y de esta forma, la comprensión de la educación desde la perspectiva de la experiencia posibilita aproximarse a lo siguiente:

Unas dimensiones del hacer pedagógico de educadoras y educadores que incorporan un saber con unas cualidades especiales, un saber no siempre fácilmente formulable, no exactamente teórico ni fácilmente teorizable, pero imprescindible como saber pedagógico; probablemente la esencia del propio saber pedagógico p. 22). 


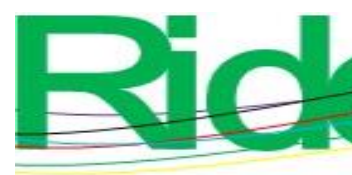

Revista Iberoamericana para la Investigación y el Desarrollo Educativo

ISSN $2007-7467$

Por experiencia, los autores citados anteriormente entienden lo vivido, los significados que otorgan a los acontecimientos, a las diversas situaciones. "Una experiencia lo es en la medida que no te deja indiferente: te implica, te afecta, te marca, te deja huella. La experiencia no es algo que sucede, sino algo que se tiene" (Contreras y Pérez, 2013, p. 24). Una muestra de este saber pedagógico adquirido por la docente como producto de su experiencia en el aula se presenta a continuación:

D-P-1: Yo siempre me he llamado como muy improvisadora, aunque yo llegue con la clase preparada a un aula, todo puede cambiar en instantes, siempre depende de la actitud de los muchachos. Para mí, la adaptación siempre la tendré como técnica; improvisar si es necesario para poder ofrecerles lo que realmente están necesitando en el momento. Yo trabajo mucho en función de las dinámicas internas en clase, a veces puedo traer la dinámica bien preparada, pero llego al salón y los jóvenes están completamente distintos a como me los imaginé, esa dinámica que yo había preparado la tengo que adaptar a las condiciones que tienen los muchachos en ese momento.

Ese improvisar en el aula es un saber que la docente ha adquirido a través de la experiencia en su diario accionar en el salón de clases. Aquí es importante señalar lo siguiente: primero, la docente prepara su clase para impartirla como corresponde, sin embargo, al llegar al aula, se da cuenta de que el clima del aula no es el adecuado para lo que ella preparó; colegimos, entonces, que el solo hecho de darse cuenta conscientemente de que su estrategia didáctica no va a funcionar es producto de una serie de fracasos que ella tuvo al empecinarse en trabajar con determinada técnica, es una situación que la marcó. Segundo, ella posee un bagaje de estrategias de enseñanza que puede poner en práctica cuando se requiere; ese saber qué y saber cuándo es producto de sus experiencias, éxitos y fracasos en su trabajo docente, estas experiencias conforman el saber pedagógico. En concordancia con el modelo de Tardif y Gauthier (2012), la docente no solo presenta de manera adecuada su discurso, sino también las razones o motivos que lo validan.

Como se ha visto, el juicio y el discurso asertivo es otra concepción del saber (Tardif y Gauthier, 2012). Enseguida se muestra un segmento narrativo que da cuenta de esta concepción: 


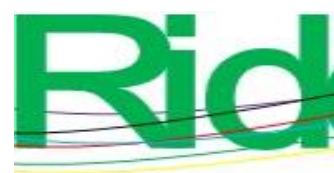

Revista Iberoamericana para la Investigación y el Desarrollo Educativo

ISSN $2007-7467$

D-P-2: Siempre procuro adaptarme a las necesidades que yo detecto en los muchachos. No todos aprendemos igual, y eso yo lo tengo muy claro desde el principio. Todos los seres humanos tenemos una tendencia para aprender de manera diferente, algunos aprendemos más enfocados en la parte visual, otros aprendemos por la parte auditiva, de igual manera otros en la parte kinestésica. Yo siempre trato de enfocarme mucho en eso, poner mucha atención en los muchachos bajo esas circunstancias.

En el caso anterior, la maestra da cuenta de un saber. En este segmento se establece un juicio cuando ella afirma que "todos los seres humanos tenemos una tendencia para aprender de manera diferente", para enseguida establecer las razones de su juicio, al mencionar las diversas maneras de aprender. Siguiendo a Tardif y Gauthier (2012), el discurso se ubica en la aserción. Y en esta concepción, solo los discursos referidos a hechos pueden ser catalogados como un saber en sentido estricto, es decir, se limita a juicios de facto y quedan fuera los de valor.

\section{Práctica profesional y docencia}

Gran parte de los docentes universitarios laboran o han laborado en el sector productivo, por lo cual sus prácticas educativas contienen elementos tanto de su formación disciplinaria como de su experiencia en el ejercicio de su profesión. De esta manera, manifiestan enfáticamente la importancia de la práctica profesional. Una docente expresa algo relacionado con esto en el siguiente segmento narrativo:

D-C-1: Los libros son muy interesantes, y trabajamos mucho en función de los libros, pero si yo tengo eso que aparece en el libro y lo viví también en la realidad, en el trabajo y desempeño profesional, trato de exponerles esas vivencias. El libro también puede quedarse limitado. Espero que los muchachos se lleven esa experiencia y que cuando ellos me hablan y me dicen: "Maestra, se acuerda de lo que nos platicó, me está pasando un poco diferente, cómo usted lo vivió, cómo le hago". Eso es lo que a mí realmente me interesa, esa experiencia que yo les pueda compartir. 


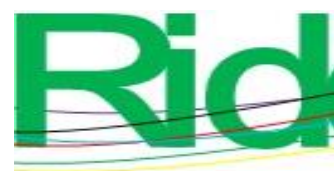

Revista Iberoamericana para la Investigación y el Desarrollo Educativo

ISSN $2007-7467$

En el segmento anterior se pone de manifiesto las ideas de Shön (1987) con respecto a la práctica profesional, en específico cuando el estudiante le comenta a la docente cómo está viviendo una experiencia sobre un caso que ella explicó en su clase y que ahora se le presenta de modo diferente. Señala Shön (1987): existen situaciones donde determinado problema no resulta inicialmente claro y no hay una concordancia entre las características de la situación y el cuerpo de teorías y técnicas disponibles para enfrentar el problema. Es frecuente, continúa Schön (1987), que en este tipo de situaciones se aluda a la expresión de "pensar como médico, o como un abogado o un directivo empresarial" para referirse al tipo de indagación que los prácticos competentes emprenden cuando recurren al conocimiento disponible para resolver aquellas situaciones de la práctica en las que la aplicación de dicho conocimiento resulta problemática. Para Schön (1987) la resolución de problemas no típicos de la práctica, las denominadas zonas de incertidumbre, tienen que ver con el arte profesional. En ese sentido, se puede reconocer la existencia de artistas profesionales capaces de dar sentido a situaciones de singularidad e incertidumbre. Sin embargo, no hay manera de decir en qué consiste ese arte; tan solo expresar que se siguen reglas que todavía no son explícitas.

La relación teoría-práctica es muy importante en el ámbito de la docencia universitaria. Al preguntarle a un docente sobre la primacía de una sobre la otra este respondió lo siguiente:

D-C-2: Las dos, creo que las dos se llevan de la mano porque se necesita experiencia para poder dar una buena clase. Parte de lo que yo he hecho lo he realizado en el sector privado dando asesorías, y después vengo y lo imparto en la docencia. Puedes tener mucha teoría pero si no tienes práctica como que no generas ese ámbito de experiencia que se requiere en un empleo.

Con relación a lo anterior, en las entrevistas se reiteró la relación teoría-práctica de diferentes maneras. Una de ellas alude a la relación entre los contenidos de los programas de estudio y la práctica en el mundo laboral. Se menciona, como tarea ineludible del trabajo docente, vincular los contenidos con el contexto empresarial y la necesidad de una actualización permanente de los planes y programas de estudio. Para el docente la realidad es el mundo del trabajo, por lo cual esa "situación real" debe trasladarse a los programas indicativos. Lo anterior sin duda es causa de tensiones, a veces las trayectorias profesionales determinan formas únicas de comprensión de los conocimientos disciplinares, los cuales se pretenden incluir tal cual en los programas educativos. En contraste, otros docentes vinculan 


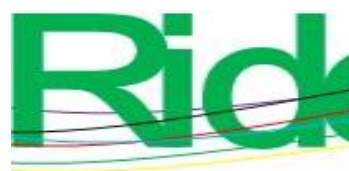

Revista Iberoamericana para la Investigación y el Desarrollo Educativo

ISSN $2007-7467$

las áreas profesionales con otros saberes para posibilitar la reflexión con las condiciones sociales de la comunidad (Escobar, 2014).

\section{La reflexión como ejercicio permanente}

Diversos autores (Erazo, 2009; Dewey, 1989; Shön, 1987; Perrenoud, 2007) señalan que la práctica reflexiva se relaciona de manera relevante con la labor docente. Esto se manifiesta en la vinculación entre acción profesional y conocimiento, e impacta de diversas maneras en los procesos de profesionalización que responden a las actuales demandas de cualificación (Erazo, 2009, p. 55). La contextualidad de la práctica reflexiva se manifiesta, de acuerdo con Shön (1987), en el carácter social de los conocimientos tácitos de los profesores a través de la noción de "prácticum". Shön (1987) afirma que, desde el punto de vista constructivista, nuestras percepciones, apreciaciones y creencias tienen sus raíces en los mundos que nosotros mismos configuramos y que terminamos por aceptar como realidad. Shön (1987), en su modelo sobre la práctica reflexiva, describe la reflexión en la acción como un proceso en el cual se clarifican situaciones de la práctica que son inciertas, singulares o conflictivas. En este tipo de prácticum se ubican aquellas situaciones en que el conocimiento profesional existente no basta ni se acomoda a cada caso. Al respecto, Perrenoud (2007) señala:

No existe acción compleja sin reflexión durante el proceso; la práctica reflexiva puede extenderse, en el sentido general de la palabra, como la reflexión sobre la situación, los objetivos, los medios, los recursos, las operaciones en marcha, los resultados provisionales, la evolución previsible del sistema de acción. Reflexionar durante la acción consiste en preguntarse lo que pasa o va a pasar, lo que podemos hacer, lo que hay que hacer, cuál es la mejor táctica, qué orientaciones y precauciones hay que tomar, qué riesgos existen, etc. (p. 30).

Para Dewey (1989), “el pensamiento reflexivo se asemeja a ese fortuito tránsito de cosas por la mente en el sentido de que consiste en una sucesión de cosas acerca de las cuales se piensa, pero se diferencia de él en que no basta la mera ocurrencia casual en una sucesión irregular de "cualquier cosa"” (p. 22). Al respecto, los docentes en su narrativa describen sus clases como algo planeado que se inicia con un planteamiento teórico acerca del tema, por 


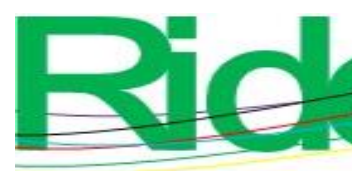

Revista Iberoamericana para la Investigación y el Desarrollo Educativo

ISSN $2007-7467$

parte del profesor, para enseguida entrar a un debate con los alumnos. Un profesor lo expresa de la siguiente manera:

D-I-2: Sí. Pues dependiendo de la unidad, dependiendo del módulo, dependiendo del tema de lo que se vaya a tratar. Por lo general se presenta lo que es un marco teórico, unos pequeños momentos de instrucción académica acerca del conocimiento de las teorías o autores para posteriormente proponer preguntas para temas de debate. Preguntas para que ellos generen el conocimiento, generen dudas y se cuestionen entre ellos. Para ya en debate irnos guiando junto con el grupo a lo que es el conocimiento o llegando junto con el grupo para que vayan aprendiendo los conceptos de la clase.

En el segmento anterior podemos observar cómo el diálogo reflexivo involucra a los estudiantes, al docente, a los contenidos de la materia y al medio que es el lenguaje, donde la pregunta reflexiva tiene una función directiva: construir significados, elaborar conceptos, interpretar, explicar (Davini, 2015). En ese sentido, de acuerdo con Dewey (1989), la reflexión no consiste tan solo en una secuencia de ideas, sino en una con-secuencia, es decir, "una ordenación consecuencial en la que cada una de ellas determina la siguiente como su resultado, mientras que cada resultado, a su vez, apunta y remite a los que la precedieron" (p. 22). Estos segmentos de un pensamiento reflexivo se originan unos de otros y se conectan entre ellos. Señala Dewey (1989): "En todo pensamiento reflexivo hay unidades definidas ligadas entre sí, de modo que acaba produciéndose un movimiento sostenido y dirigido hacia un fin común" (p. 22).

Las reflexiones de los docentes en torno a sus prácticas de enseñanza y a los aprendizajes de los alumnos nos llevan a considerar que ciertos profesores poseen diversas concepciones teóricas que ponen en juego en su actuar docente. Al respecto, un profesor expresó:

D-P-3: Desde mi punto de vista me gusta utilizar lo que es, más que nada, la metacognición, para que ellos comprendan lo que es el conocimiento, más que aprender conceptos, más que aprender fechas, nombres. Que realmente entiendan el pensamiento y lo puedan aplicar a sus vidas y hacerlo propio. Más que cuestionarios, más que con cuadros, con herramientas muy tradicionales, poderlos aplicar de una manera más vivencial el conocimiento. 


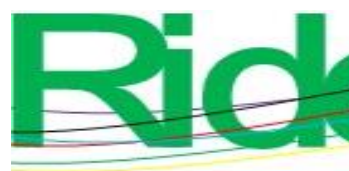

Revista Iberoamericana para la Investigación y el Desarrollo Educativo

ISSN $2007-7467$

La reflexión del docente sobre su práctica es un ejercicio permanente que debe de ejercer con miras a una mejora y fortalecimiento de su quehacer en el aula. Esto da pie a que el profesor ejerza la docencia construyendo nuevos saberes que le posibiliten enfrentar diversas situaciones en su ejercicio cotidiano en el aula. Perrenoud (2007) afirma que la acción en la práctica reflexiva lleva implícita una representación; se da por hecho que el sujeto tiene conocimiento de lo que hace y, por lo tanto, puede preguntarse por los móviles, las formas y los efectos de su acción. Perrenoud (2007) también se pregunta: “¿En qué se convierte la reflexión cuando su objeto desaparece, cuando su propia acción escapa al control del actor?” (p. 137). Las prácticas realizadas por distintos profesionales, llámese un ingeniero o un médico, la racionalidad implícita en determinado proceso, sea un cálculo en el caso del ingeniero o una auscultación en el caso de un médico, hace que los procedimientos llevados a cabo en la acción queden ocultos por "el carácter parcialmente inconsciente de la actividad" (Perrenoud, 2007, p. 137). La secuencia de ideas llevada a cabo en una reflexión sostenida en los conocimientos de lo práctico hace que el flujo inconsciente de unidades se manifieste espontáneamente. Perrenoud (2007) denomina a lo anterior inconsciente práctico. El autor lo vincula con los trabajos relacionados con la acción prerreflexionada basados en la entrevista de explicitación: Vermersch (1994) Vermersch y Maurel (1997); la teoría piagetiana del inconsciente práctico y los esquemas: Piaget (1973, 1974), Vergnaud (1990, 1994, 1995, 1996), y la teoría del habitus vinculada a la obra de Bourdieu (1972, 1980; citado en Perrenoud, 2007, p. 138). De igual forma, Perrenoud (2007) observa una convergencia con los trabajos sobre la transferencia y las competencias que aluden a los procesos de movilización de recursos cognitivos guardados en el inconsciente. Este proceso, señala Perrenoud (2007):

No es otra cosa que el funcionamiento del habitus que, enfrentado a una situación, lleva a cabo una serie de situaciones mentales que garantizará la identificación de los recursos pertinentes, su transposición eventual y su movilización orquestada para producir una acción adecuada (p. 138).

El habitus, entonces, siguiendo a Perrenoud (2007), sería el conjunto de esquemas o experiencias que un sujeto utiliza en determinada situación y que permite resolver problemas de la misma manera. Una docente expresa cómo a partir de su experiencia profesional ella vive la docencia: 


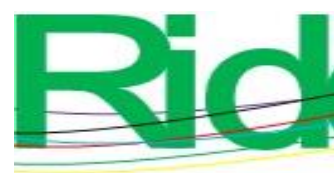

Revista Iberoamericana para la Investigación y el Desarrollo Educativo

ISSN $2007-7467$

D-P-2: Siempre lo que sé, lo que he vivido y lo que he experimentado lo voy a poner en el escritorio de la docencia. Mis muchachos ya saben que yo soy como muy descriptiva - ellos me llaman contadora de historias-; la verdad lo que les estoy contando es mi experiencia, es mi vivencia, lo que me ha tocado vivir profesionalmente y siempre lo hago con la intencionalidad de dejarles algo. Decirles: allá afuera está pasando esto, así se viven las cosas, esto que estamos viendo en lo teórico, aquí en el libro, se manifiesta de esta manera en la realidad. Siempre voy a procurar hacer ese enlace.

La especificidad de la disciplina está en la base del proceso de enseñanza implementado por el profesor. En este ejemplo, la experiencia laboral de la docente tiene una gran influencia. Ella relaciona los contenidos de la materia con los casos que vive o vivió en su experiencia laboral. La planeación está vertebrada por estas experiencias que el docente vincula con la teoría y las actividades o procesos llevados a cabo por profesionales. Con respecto a la relación teoría-práctica, un docente expresa:

D-D-1: Mi área, que es la criminalística, como en toda carrera se presentan cargas teóricas, las cuales son muchas veces criticadas por los alumnos, ya que se quejan de la teoría. Uno lo que hace es insistirles en que reflexionen en cuestión de que la teoría es importante para cualquier cosa; lo que pasa es que algunos maestros no abordamos bien la teoría, de tal manera que no hacemos que al alumno le guste. La teoría es el marco de referencia básico para que podamos irnos a la práctica. En la práctica se aterriza la teoría y es hacerla valida en cuanto al qué y el para qué, si queda nada más en teoría nos quedaremos en el qué.

En este segmento es posible observar un ejercicio de reflexión sobre la acción, en este caso la crítica de los estudiantes a la teoría. Al respecto, el docente reflexiona para comprender e integrar lo que ha sucedido. En ese línea, Perrenoud (2007) expresa: "Entonces, reflexionar no se limita a una evocación, sino que pasa por una crítica, un análisis, un proceso de relacionar con reglas, teorías u otras acciones, imaginadas o conducidas en una situación análoga" (p. 33). 


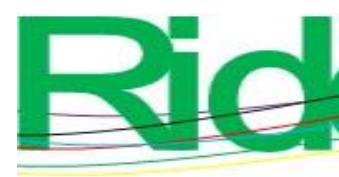

Revista lberoamericana para la Investigación y el Desarrollo Educativo ISSN $2007-7467$

\section{Discusión}

En las entrevistas los docentes expresaron sus conocimientos, experiencias y saberes en concordancia con el modelo de Tardif y Gauthier (2012), que manifiesta los elementos que se deben tomar en cuenta en la investigación de los saberes docentes. Las certezas subjetivas, los juicios y argumentos presentados en torno a sus prácticas pedagógicas se manifestaron con una racionalidad en el pensar y el hablar, con la cual exponen las razones que orientan su práctica. Consideramos que en esta recuperación de saberes docentes radica una de las fortalezas de nuestro trabajo. Desde ese punto de partida, en el análisis fue posible construir las categorías que abordan el panorama en torno a las prácticas pedagógicas universitarias. Por otra parte, desde su inserción en el ámbito laboral de la docencia universitaria, el perfil del docente adquiere ciertas peculiaridades dependiendo de su experiencia profesional, la cual define las formas de concebir la disciplina y con ella la enseñanza. De acuerdo con Escobar (2014):

Se entiende la disciplina como un sistema de relaciones simbólicas que puede incluir los lugares físicos de trabajo y las relaciones sociales propias de esos ámbitos, los lenguajes, saberes, prácticas, hábitos, actitudes, modos de comportamiento, relaciones de poder y de autoridad, incluyendo atributos personales como la disciplina, la exigencia, la responsabilidad, la creatividad y la proactividad (p. 299).

En las diversas narrativas los docentes reconstruyen sus actos docentes en un ejercicio reflexivo. Y desde allí se manifestaron los saberes disciplinarios como ejes de su enseñanza, mientras que los saberes pedagógicos permanecen al margen, hasta que el entrevistador interroga al respecto, y se vuelven presentes en los recursos y saberes experienciales. Con ellos los profesores en el ámbito de su acción pedagógica desarrollan estrategias de acción que facilitan los procesos de enseñanza y aprendizaje. No obstante, el saber profesional tiene una mayor legitimidad en el imaginario universitario.

En las entrevistas los profesores universitarios describen prácticas pedagógicas con una amplia gama de conocimientos y saberes, los cuales se caracterizan por su complejidad. En esa tesitura, las investigaciones de las ciencias cognitivas han resultado insuficientes para explicar las diversas problemáticas (Altet, 2012). De ahí la pertinencia del modelo reflexivo (Dewey, 1989; Tardif, 2009; Perrenoud, 2007), que posibilita entender las acciones del docente en 


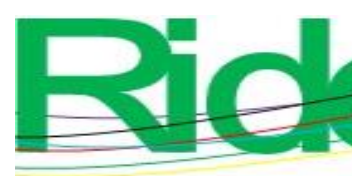

Revista Iberoamericana para la Investigación y el Desarrollo Educativo

ISSN $2007-7467$

situaciones inciertas, singulares o conflictivas. En las narraciones, asimismo, los docentes manifestaron ideas que exponen la reconstrucción de la experiencia en el aula y la presencia de saberes pedagógicos fruto del proceso reflexivo de su trabajo docente. Esto en coincidencia con Tardif (2009), Perrenoud (2007) y Shön (1978). Un hallazgo importante es que los docentes no interpretan su experiencia y sus actos educativos desde las teorías educativas. Así, llaman improvisar a un saber pedagógico indiscutible; de ahí la necesidad de identificar e interpretar estas prácticas pedagógicas para convertirlas en saberes explícitos y recuperarlos para la práctica (Contreras y Pérez, 2013).

En su trabajo en el aula los profesores de la universidad dan primacía a sus conocimientos disciplinares. Lo cual motiva que las interacciones se estructuren en torno al discurso de la profesión. Sin embargo, los estilos de enseñanza son heterogéneos y a veces diametralmente opuestos dependiendo del programa educativo de que se trate, de tal forma que no es lo mismo una clase de derecho que generalmente se centra en la práctica discursiva del docente, que una de psicología donde los profesores generan diversas dinámicas y dispositivos para el aprendizaje.

No obstante, no se deben de realizar juicios a priori, estigmatizando una enseñanza centrada en el discurso del docente. Muchas veces dentro de este discurso se realizan descentraciones múltiples bien sustentadas por el profesor, que interesan y fomentan los aprendizajes en los estudiantes. De ahí la necesidad de realizar investigaciones que incluya la observación de la labor docente en las aulas, una de las principales limitaciones de este trabajo. Sin duda coincidimos con Contreras y Pérez (2013) cuando señalan: "Queremos comprender y compartir experiencias de maestros universitarios, como acontecimientos abiertos a nuevas significaciones; aportar a la pedagogía de la educación superior, como saber que se nutre de vivencias docentes, que cobran sentido desde sus voces" (p. 342). 


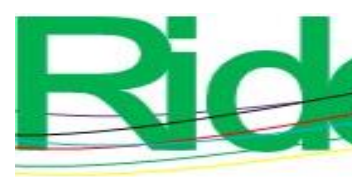

Revista Iberoamericana para la Investigación y el Desarrollo Educativo

ISSN $2007-7467$

\section{Conclusiones}

La interpretación de las narrativas de los profesores en torno a sus prácticas pedagógicas desde la perspectiva reflexiva permite dilucidar que las prácticas pedagógicas de los profesores son consecuencia de un conjunto de saberes producto de la experiencia cotidiana del profesor en el aula y en el ámbito universitario, con profundas huellas de la formación profesional. Asimismo, las prácticas de enseñanza tienen una impronta de la historia personal y las vivencias sociales, las cuales se vinculan con los contenidos y objetivos de aprendizaje.

El profesor que centra su enseñanza en los saberes disciplinarios intenta reproducir las prácticas laborales en el aula, donde la disciplina enmarca las acciones en una formación para el trabajo que concibe el mundo laboral como la realidad. La especificidad de la disciplina está en la base del proceso de enseñanza implementado por el docente. De esta manera, la planeación está vertebrada por las experiencias laborales que el docente vincula con la teoría y las actividades o procesos llevados a cabo por profesionales. Sin embargo, los estilos de enseñanza son diversos, y así también existen docentes preocupados por el aprendizaje de los estudiantes que se manifiesta en diversos aspectos, tal y como detectar el clima de la clase antes de iniciar actividades, escoger en la inmediatez de la acción el tipo de estrategia adecuada para determinado ambiente de aprendizaje y fomentar el diálogo reflexivo con los estudiantes.

En este entorno es posible percibir cómo el docente genera saber pedagógico en su accionar en el aula a partir de sus experiencias vividas. Los saberes pedagógicos de los docentes universitarios develados en este trabajo permiten dilucidar que estos son producto de una práctica reflexiva. En ese sentido, la experiencia es concebida no como la realización de una tarea rutinaria, sino como una acción positiva o negativa que deja una huella con un significado que se ha de valorar en una reflexión sobre la acción. La recuperación de los saberes pedagógicos presentes en la narrativa de los profesores universitarios permitió avizorar un territorio inexplorado, pues, en el ámbito de este nivel, no es valorado como un saber indiscutible que se desplaza en el entramado de los saberes disciplinarios. Concebir la docencia desde una perspectiva reflexiva es retomar la idea de promover una racionalidad de la práctica que permita al docente universitario resolver sus problemas cotidianos de una 


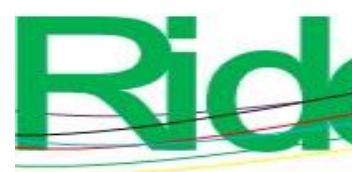

Revista Iberoamericana para la Investigación y el Desarrollo Educativo

ISSN $2007-7467$

manera profesional. Reflexionar la experiencia que se vive, exponerla a través de narraciones, se constituye en una tarea actual que los docentes deben realizar.

\section{Referencias}

Altet, M. (2012), La competencia del maestro profesional o la importancia de saber analizar las prácticas. En Paquay, L., Altet, M., Charlier, P. y Perrenoud, P. (coords.), La formación profesional del maestro. Estrategias y competencias. México: FCE.

Beuchot, M. (2008). Perfiles esenciales de la hermenéutica. México: FCE.

Beuchot, M. (2016). Hechos e interpretaciones. Hacia una hermenéutica analógica. México: FCE.

Contreras, J. y Pérez, N. (2013). Investigar la experiencia educativa. Madrid, España: Morata.

Davini, M. (2015). La formación en la práctica docente. Argentina: Paidós.

Dewey, J. (1989). Cómo pensamos. Nueva exposición de relaciones entre pensamiento y proceso educativo. España: Paidós.

Escobar, C. (2014). Saberes de profesores universitarios. Una fuente de saber pedagógico. En Londoño, G. (coord.), Docencia universitaria. Sentidos, didácticas, sujetos y saberes. Colombia: De La Salle Ediciones.

Erazo, M. (2009). Práctica reflexiva como estrategia de desarrollo profesional: presencia y estructura en reuniones docentes. Educación y educadores, 12(2), 47-74. Recuperado de http://www.redalyc.org/artículo.oa?id=83412219004.

Fierro, C., Fortoul, B. y Rosas, L. (2002). Transformando la práctica docente. Una propuesta basada en la investigación-acción. México: Paidós.

Goetz, J. y LeCompte, M. (1988). Etnografía y diseño cualitativo en investigación cualitativa, Madrid: Morata.

Jiménez J. y Rendón, M., (2014). Didáctica universitaria. En Londoño, G. (coord.), Docencia universitaria. Sentidos, didácticas, sujetos y saberes. Colombia: De La Salle Ediciones.

Londoño, G. (2014). Prácticas de enseñanza y saber pedagógico de profesores universitarios. En Londoño, G. (coord.), Docencia universitaria. Sentidos, didácticas, sujetos y saberes. Colombia: De La Salle Ediciones. 


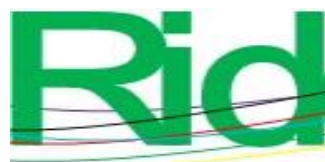

Revista lberoamericana para la Investigación y el Desarrollo Educativo

ISSN $2007-7467$

Lüdke, M. (2010). El trabajo y el saber docente: nuevos y viejos desafíos. En Tenti, E. (comp.), El oficio de docente: vocación, trabajo y profesión en el siglo XXI. México: Siglo XXI.

Paquay, L., Altet, M., Charlier, P. y Perrenoud, P. (coords.) (2012). La formación profesional del maestro. Estrategias y competencias. México: FCE.

Perrenoud, P. (2007). Desarrollar la práctica reflexiva en el oficio de enseñar. Ciudad de México, México: Colofón.

Roldán, Y. (2016). Una mirada analógica a las representaciones sociales del docente en torno a la inclusión educativa. En Monroy, F. (coord.), Temas de formación docente. México: Publicaciones Académicas CAPUB.

Schön, D. ( 1987). La formación de profesionales reflexivos. Madrid, España: Paidós.

Tardif, M., Lessard, C. y Lahaye, L. (1991), Os professores em face do saber: Esboco de uma problemática do saber docente. Teoria \& Educacao, (4), 215-233.

Tardif, M. (2009). Los saberes del docente y su desarrollo profesional. Madrid, España: Narcea.

Tardif, M. y Gauthier, C. (2012). El maestro como actor racional: racionalidad, conocimiento, juicio. En Paquay, L., Altet, M., Charlier, P. y Perrenoud, P. (coords.), La formación profesional del maestro. Estrategias y competencias. México: FCE. 